\title{
Conception d'un BioMicroRobot autonome
}

\author{
Réjean Fontaine, Martin Otis, Martin Jarry, Hicham Semmaoui, Marie-Ève Gosselin, Patrice Masson, \\ Hugues Ménard
}

\begin{abstract}
Résumé-Cet article présente les avancements technologiques pour la conception théorique et pratique d'une architecture élémentaire et minimale d'un BioMicroRobot (BMR) hexapode autonome. L'autonomie minimale dépend du système de gestion des événements globaux, régionaux et locaux. Cette gestion est avant tout perçue par deux types de récepteurs: les extérocepteurs internes (vision) et ceux externes (système de repérage et de détection de l'environnement). La planification des événements dans un environnement confiné repose sur un système complexe qui incorpore un microcontrôleur d'architecture RISC, un système de propulsion bio-inspiré indépendant qui agit comme un pilote, un système d'alimentation et de communication et finalement un détecteur de proximité qui agit à titre d'extérocepteurs internes. L'avant dernière section présente un procédé de fabrication de la structure complète du BMR qui peut supporter une hiérarchie hybride à deux niveaux avec la logique floue.
\end{abstract}

Index Terms-IPMC, PWM, Nafion, biomicrorobot, CPG.

\section{INTRODUCTION}

L a microrobotique a refait surface récemment avec l'arrivée

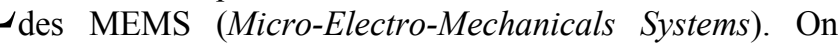
note beaucoup d'intérêt dans la micromanipulation de microorganismes, dans l'assemblage de micromoteur et dans l'exploration des milieux fortement confinés. Ce domaine est une science en émergence qui lie les technologies développées par la microélectronique et par la robotique [1]. Le projet, développé au Groupe de recherche en appareillage médical de Sherbrooke (GRAMS), est au centre d'une équipe multidisciplinaire qui regroupe plusieurs acteurs en chimie, en physique, en électronique et en mécanique, possédant les connaissances techniques nécessaires à l'accomplissement des travaux de recherche en biomicrorobotique. Ce domaine implique la conception et la fabrication de microrobots, voire de nanorobots pouvant se déplacer dans un fluide biologique pour des applications biomédicales. Ceux-ci doivent être capables d'effectuer certaines réparations du corps humain et

R. Fontaine, M. Otis, M. Jarry, et H. Semmaoui sont du Département de génie électrique et de génie informatique, Université de Sherbrooke, Sherbrooke, J1K2R1, CANADA; courriel: Martin.Otis@USherbrooke.ca, Martin.Jarry@USherbrooke.ca,_Hicham.Semmaoui@USherbrooke.ca, Rejean.Fontaine@USherbrooke.ca.

M.-E. Gosselin est du Département de physique, Université de Sherbrooke, Sherbrooke, J1K2R1, CANADA; courriel: MarieEve.Gosselin@USherbrooke.ca

P. Masson est du Département de génie mécanique, Université de Sherbrooke, Sherbrooke, J1K2R1, CANADA; courriel: patrice.masson@gme.usherb.ca.

H. Ménard est du Département de chimie, Université de Sherbrooke, Sherbrooke, J1K2R1, CANADA; courriel: Hugues.Ménard@USherbrooke.ca. même d'y manipuler des cellules. Ce domaine en est encore qu'à ses débuts et se poursuivra sur plusieurs années. Par conséquent, il est envisageable de tirer profit de la miniaturisation des circuits et des composantes électroniques pour diminuer la dimension du BMR

Les travaux du GRAMS se concentrent sur la conception théorique et la fabrication d'un BioMicroRobot (BMR) hexapode autonome de l'ordre du millimètre basé sur un ionomère à échange d'ions et sur des céramiques d'alumine. Sa conception a nécessité le développement de circuits intégrés basé sur la technologie CMOS P18 pour le contrôle dudit BMR.

Les travaux de recherche actuellement en cours explorent différents axes, dont la conception de nouveaux mécanismes et dispositifs de propulsion millimétriques et micrométriques, l'organisation spatiale, l'intelligence artificielle, et la manipulation d'objets micrométriques [2]. Un des objectifs consiste à la commande des pattes selon le dispositif de vision ce qui implique plusieurs contraintes dont un contrôle adéquat et un faible volume afin de pouvoir l'implanter dans le corps humain.

Le développement du BMR nécessite d'utiliser et de valider plusieurs algorithmes de contrôle. La conception de ces algorithmes est critique, car de cette manière, il sera possible de trouver celui qui permet d'obtenir un déplacement optimal $\mathrm{du}$ BMR et une amélioration de la puissance de consommation. Comme plusieurs algorithmes doivent être expérimentés, il est donc primordial que le circuit intégré utilise une architecture qui soit souple et reprogrammable.

L'implantation à l'intérieur du corps humain nécessite d'utiliser des matériaux biocompatibles et, ce faisant, il ne sera pas possible de relier le circuit par un lien physique. Étant donné que les piles d'alimentation existantes actuellement sont encore trop imposantes, ce BMR devra être alimenté par un système externe; ce qui entraîne une limitation de la puissance disponible. De plus, la programmation du robot doit être assurée par un lien de communication sans fil à haut rendement; ce qui nécessitera un lien à faible débit. Comme la puissance et les communications seront acheminées via ce lien externe, il est important de sélectionner une technologie ayant une faible consommation d'énergie et de choisir un protocole de communication pouvant être transmis via un lien RF (Radio-Fréquency) adéquat.

Le volume du premier BMR contenant le circuit intégré qui servira à démontrer sa faisabilité sera de moins de $4 \mathrm{~mm}$ X 6 $\mathrm{mm} X 4 \mathrm{~mm}$. De plus, ce volume sera partagé avec des composantes RF et des circuits passifs. Par conséquent, la 
dimension à respecter pour le circuit de contrôle doit être minimale.

Comme les contraintes de dimensions, de puissance consommée par le circuit, de flexibilité au niveau de l'entrée des données et le placement de ce circuit sont critiques, l'utilisation de composants commerciaux ne sont pas possibles. Il est donc approprié de concevoir l'ensemble des circuits requis à l'Université. Dû à la diminution de la dimension des transistors, l'intégration des circuits intégrés à large échelle est appropriée lorsque la puissance est à optimiser tel qu'en biomicrorobotique.

\section{SYSTĖMES DE CONTRÔLE DU BMR}

Le circuit intégré du BMR est basé sur la conception d'un microcontrôleur conçu sur une architecture RISC de huit bits avec un décodeur Manchester pour des fins de télécommunication. Comme le signal d'entrée se propage à travers un port série, un convertisseur série/parallèle a été implémenté pour télécharger les instructions dans la mémoire du BMR sur une technologie CMOS P18 disponible à travers la Société Canadienne de Micriélectronique (SCM). Le signal de sortie pour le contrôle des pattes est transmis par un port parallèle de huit bits. Lors de l'opération de programmation du BMR, l'horloge du système est extraite du décodeur Manchester - qui extrait son horloge à partir du signal RF acquis (voir section B), - cependant, pour des fins d'expérimentations, les données et l'horloge peuvent être acheminées par une source externe [3], [4] et [5]. Pour le premier prototype, l'horloge sera extraite du décodeur par le même lien inductif qui génère la source d'alimentation du BMR. L'utilisation d'une architecture RISC avec un accumulateur est bien adaptée pour ce type d'application puisque la circuiterie est réduite comparativement aux autres architectures. De plus, la vitesse de l'horloge du système est limitée à $1 \mathrm{MHz}$ pour limiter la puissance de consommation.

\section{A. Système de propulsion bio-inspiré}

Les systèmes de propulsion des microrobots sont très variables. Certaines équipes anticipent l'utilisation de champs magnétiques oscillant afin de générer un mouvement au MicroRobot [6], [7]. Cette approche est excellente pour effectuer un déplacement grossier du robot mais comportes des inconvénients pour des déplacements fins. L'approche du présent BMR utilise un système de contrôle inspiré de la biologie d'un insecte à six pattes. Les travaux actuels sont divisés en trois parties. La première vise à trouver un moyen de propulsion original. La deuxième partie porte sur la fabrication et la caractérisation de l'actionneur. Elle inclue le développement d'un nouveau procédé de fabrication chimique pour réaliser les micropattes du BMR. Finalement, la troisième porte sur l'étude d'un générateur de patron centralisé autonome.

Les actionneurs électromécaniques (micropattes) sont composés d'un ionomère perfluorosulfonique à échange d'ions de type cationique et du platine, soit un IPMC (Ionic Polymer Metal-Composite) Nafion-Pt. Leurs formes géométriques sont quasi-cylindriques ou tubulaires. Le procédé est effectué dans le laboratoire du département de chimie réservé à l'Institut des Matériaux et des Systèmes Intelligents (IMSI). La disposition des électrodes, sur le polymère, leur attribue 2.5 degrés de liberté (DOF), ce qui confère au robot $15 \mathrm{DOF}$, contrairement à un insecte qui en possède plus de 18 . Le procédé de coupe des quatre électrodes est effectué à l'aide d'un laser excimer du Groupe d'acoustique et de vibrations de l'Université de Sherbrooke (GAUS) (Fig. 1).

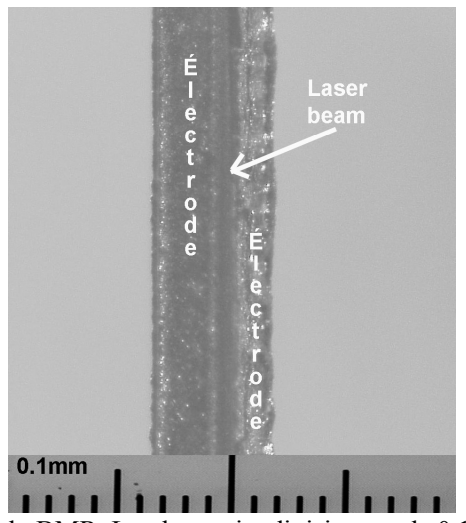

Fig. 1 Micropatte du BMR. La plus petite division est de $0.1 \mathrm{~mm}$.

Les caractérisations morphologique et mécanique sont exécutées par la suite. La caractérisation morphologique comprend l'analyse d'images transversales de l'actionneur prise par microscopie électronique (SEM). Ces images permettent de valider la déposition du platine sur et à l'intérieur du ionomère. Quant à la caractérisation mécanique, elle est réalisée avec un laser vibromètre du GAUS afin de mesurer les déplacements millimétriques de l'actionneur en fonction de la tension efficace générée par la PWM, des fréquences imposées et du courant électrique induit dans l'IPMC.

Enfin, un système de contrôle bio-inspiré, selon les principes de déplacement d'un insecte, contenant un CPG (Central-Pattern Generator), est utilisé pour effectuer le contrôle des pattes (Fig. 2).

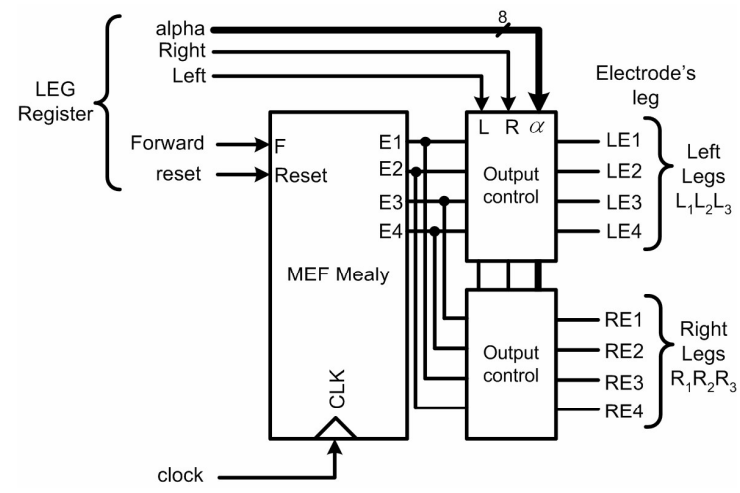

Fig. 2 Pilote de contrôle de la direction basé sur un CPG.

L'algorithme, codé en VHDL produit un déplacement linéaire et curviligne autonome des six pattes, selon une machine à états finis (MEF) de Mealy et une modulation de 
type PWM. L'utilisation d'une modulation de type PWM est requise car le microcontrôleur d'architecture RISC conçu pour cette application ne supporte pas plusieurs niveaux de tensions à la sortie du port parallèle. Puisque l'IPMC peut être modélisé par des éléments passifs comme les capacités et les résistances, l'utilisation de la PWM permet d'ajuster le niveau de la tension efficace. De cette manière, le rapport de cycle peut contrôler l'angle de courbure [8], [9] et [10].

\section{B. Système de communication et d'alimentation}

L'alimentation autonome des BioMicroRobots (BMR) constitue un défi de taille. Afin de fournir l'énergie nécessaire au BMR, l'utilisation d'un lien inductif basé sur une bobine d'une géométrie spéciale appelé bobine d'Helmholtz pilotée par un amplificateur de puissance classe $\mathrm{E}$ et un modulateur AM (code Manchester) est nécessaire [11], [12], [13], [14] et [15]. De son côté, le BMR possèderait une minuscule bobine (antenne réceptrice), et un circuit électronique simple de traitement du signal capable de récupérer l'énergie de la porteuse (Fig. 3).

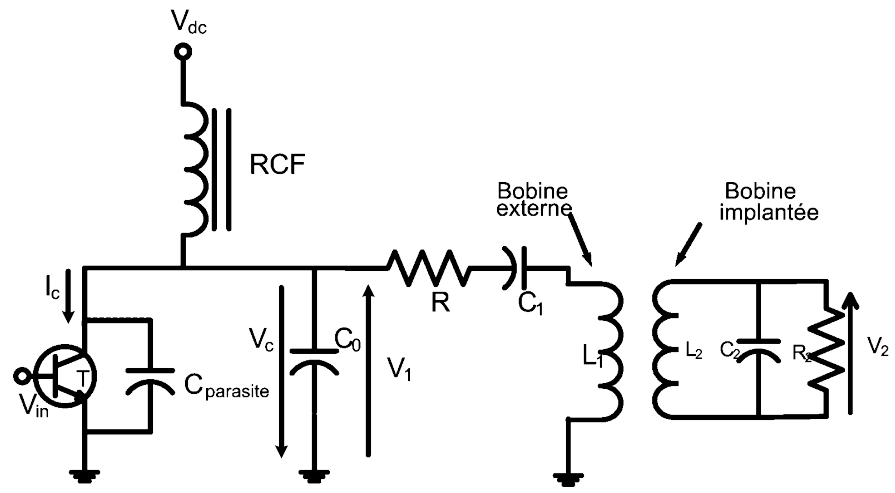

Fig. 3 Topologie de la classe E avec un lien inductif pour l'alimentation du BMR.

Le lien est utilisé avec une configuration dite sérieparallèle, qui reste la meilleure quand l'objectif est d'avoir un gain de tension constant même avec une charge variable. La syntonisation utilisée est dite double syntonisation: elle va permettre d'avoir un grand rendement de puissance, contrairement à la dé-syntonisation qui sacrifie le rendement au détriment de la stabilité [16].

La configuration série utilisée au primaire s'adapte bien à l'utilisation d'un amplificateur de puissance classe E pour piloter le lien avec un rendement en puissance très significatif. Le contrôle du BMR est assuré par un simple modulateur AM, suivant les mots de commande en code Manchester. La démodulation utilise un circuit original utilisant un seul transistor et consommant une très faible puissance.

\section{Système de détection de proximité}

Le concept général du micro-sonar repose sur la génération d'une onde ultrasonore par un actionneur piézoélectrique. La proximité des objets environnants sera déterminée en fonction de la phase du signal capté par un second piézoélectrique, résultant de la vibration de l'onde réfléchie. Une tension de l'ordre de quelques centaines de microvolts doit être présente sur le récepteur afin d'être mesurée par les dispositifs du microrobot. Avec la méthode des éléments finis, les logiciels Atila, Patran et Nastran ont été utilisés afin de déterminer une configuration optimale de l'actionneur et du capteur. En première approximation, la biocompatibilité des matériaux a été négligée afin de simplifier les simulations. Ces dernières se sont effectuées avec un support d'acier, des électrodes d'or et un actionneur (capteur) de PZT-5a.

Plusieurs géométries ont été testées, dont un empilement de deux couches piézoélectriques alternées par trois électrodes. Avec des membranes piézoélectriques de $300 \times 300 \times 15$ microns, la résonance se situait toujours autour de $2 \mathrm{MHz}$. L'optimisation de cette approche a démontré qu'une résonance à environ $200 \mathrm{kHz}$ était possible avec des dimensions de 30 x 500 × 3,5 microns. Cette configuration, prometteuse, a donc été conservée pour les recherches ultérieures. L'étape de la simulation du rayonnement des ondes dans l'eau est en cours afin de déterminer la configuration optimale [2].

\section{ProcédÉ de FABRICATION TRIDIMENSIONNELLE DU BMR}

La difficulté majeure des BMR est de concevoir une structure tridimensionnelle. Plusieurs approches sont actuellement en cours pour établir un moyen de joindre les actionneurs tubulaires ou en fibres (quasi-cylindrique) et le microcontrôleur à la structure du BMR. La méthode envisagée consiste à fabriquer les micropattes indépendamment du reste de la structure du robot à l'aide d'un procédé décrit à la section 2.A. La difficulté de cette méthode est de coller les pattes sur le corps du BMR sans sécher le IPMC au contact de l'air. Ce problème est actuellement sous investigation.

Le corps du robot peut être réalisé avec plusieurs types de matériaux comme la céramique et le Nafion. Le premier qui est investigué dans ces travaux de recherche est la céramique d'alumine. Elle offre plusieurs avantages en ce qui concerne sa manipulation et la réalisation des liens électriques. D'ailleurs, il est possible d'y déposer du platine par évaporation E-beam et de fabriquer les liens électriques avec le laser excimer. Une méthode simple utilise plusieurs couches de lamelles de céramique pour fabriquer le corps du BMR. Des trous rectangulaires peuvent être réalisés dans les premières lamelles du matériel pour y insérer le microcontrôleur. Les autres couches inférieures serviraient au support des micropattes et des liens électriques.

\section{Commande D'Un BMR AVEC la logique Floue}

Plusieurs éléments sont à considérer lors du choix d'une technique de commande. Dans notre cas, les principaux sont la limitation de la taille du circuit et l'intégration de plusieurs niveaux de raisonnement. Ces raisonnements peuvent être réactif (modifier la trajectoire) et délibératif (planification). Considérant ces éléments, la technique de la logique floue est un choix justifié, car il est possible de conserver un code limité pour le contrôle et une taille de mémoire compacte [17]. 
L'architecture floue étudiée privilégie une approche comportementale hybride.

Les comportements sont ajustés en temps réel par l'utilisation de poids relatifs variant dynamiquement selon les entrées du système. Comme la taille du circuit est critique pour un microrobot, il est impératif de diminuer autant que possible le nombre de règles de logique tout en s'assurant que le modèle puisse rencontrer les objectifs prédéfinis. Ainsi, à la limite, le nombre de règles suit l'équation $m^{n}$ où $n$ est le nombre de variables et $m$ le nombre de termes. C'est pourquoi, dès l'étape de conception du modèle, il faut regarder les alternatives pour diminuer le nombre de règles [18]. Aussi, il faut s'assurer de bien définir les tâches que le robot doit accomplir afin de choisir la bonne architecture [19].

Concernant les différentes approches en logique floue, celle apportée par Brooks qui consiste à utiliser une architecture par comportements, où ceux-ci fonctionnent en parallèle, est robuste [20], [21]. Celle-ci a permis la conception de plusieurs robots au cours des années subséquentes. Cependant, dans son approche des niveaux hiérarchiques, celui-ci préconise un modèle sans représentation qui stipule que le monde est à son meilleur modèle [21].

Cette approche n'est pas applicable considérant la complexité de l'environnement du BMR. Une évolution de l'approche de Brooks est une approche hybride développé par Saffiotti, dont la couche supérieure comprend une modélisation de l'environnement et une planification de la tâche à accomplir; alors que la couche inférieure inclut les comportements à suivre par le microrobot [22]. Ainsi, le niveau supérieur comprenant les modules de planification et de modélisation ne seront pas incorporés à même le BMR. Ils seront plutôt reliés par l'intermédiaire du lien RF dans le but de diminuer la taille du circuit qui sera intégré au robot.

\section{CONCLUSION}

Cet article présente une avenue possible pour la conception et le développement complet d'un BioMicroRobot dédié à des applications médicales. Entre autres, un circuit intégré incluant un décodeur Manchester, un convertisseur série/parallèle et un microcontrôleur pour un BMR est revu. Ce circuit reçoit et traite l'algorithme de contrôle pour le déplacement des six micropattes du BMR. L'architecture RISC pour le microcontrôleur a été choisie pour la flexibilité de sa programmation, la consommation réduite en puissance et ses dimensions d'implémentations. Le BMR autonome nécessite un lien inductif (RF) pour les télécommunications, pour son alimentation et pour son horloge interne. La conversion série/parallèle est utilisée lors des télécommunications et pour le chargement de la SRAM. Finalement, l'utilisation d'une machine à états finis et de la PWM pour actionner les micropattes permettent de déplacer le BMR dans toutes les directions de la même manière qu'un insecte à six pattes.

\section{REFERENCES}

[1] N. Chaillet, La microrobotique, un nouveau champ d'investigation pour la robotique, J'automatise, p.31-36, fév. 1999.

[2] M. Otis, M. Jarry, M.-E. Gosselin, R. Fontaine, P. Masson, Conception et fabrication d'un microrobot avec des ionomères perfluorosulfoniques à échange d'ions, $2^{\text {ième }}$ colloque de l'IMSI, Université de Sherbrooke, Canada, Sherbrooke, 2002.

[3] M. Jarry, M. Otis, H. Semmaoui, G. Haché, R. Fontaine, "Core Control Circuit for SubmillimiterRobot," The 25th Silver Anniversary International Conference of the IEEE Engineering in Medicine and Biology Society, p.3427-3430, Cancun, Mexico, sept. 2003.

[4] M. Jarry, M. Otis, H. Semmaoui, R. Fontaine, "Microcontroller architecture for autonomous BioMicroRobots Displacement Control," NEWCAS'2003 proceeding, ReSMiQ's first Northeast Workshop on Circuits and Systems, p. 73-76, Montréal, Canada, juin 2003.

[5] M. Jarry, Conception d'un microcontrôleur avec la technologie CMOS P18 pour un BioMicroRobot, mémoire de maîtrise, Université de Sherbrooke, Sherbrooke, Canada, 2004.

[6] K. Ishiyama, M. Sendoh, A. Yamazaki, K. I. Aeai, "Swimming micromachine driven by magnetic torque, "Proc. of the 3rd European Conference on Magnetic Sensors and Actuators, vol. 91 - no. 1-2, p. 141-144, juin 2001.

[7] J-B. Mathieu et al., "MRI Systems as a Mean of Propulsion for a Microdevice in Blood Vessels", Proc. EMBS, p. 3419-3422, Cancun, Mexico, Sept. 2003.

[8] M. Otis et al., "Development of an Hexapod BioMicroRobot with Nafion-Pt IPMC Microlegs," Proc. EMBS, p. 3423-3426, Cancun, Mexico, sept. 2003.

[9] M. Otis, M. Jarry, R. Bernier, R. Fontaine, H. Ménard, P. Masson, “3D Modelization of an hexapod BioMicroRobot propulsion system with IPMC actuators," NEWCAS'2003 proceeding, ReSMiQ's first Northeast Workshop on Circuits and Systems, p. 65-68, Montréal, Canada, juin 2003.

[10] M. Otis, Dévelopement d'un système de propulsion pour un BioMicroRobot Hexapode avec un ionomère perfluorosulfonique, mémoire de maîtrise, Université de Sherbrooke, Sherbrooke, Canada, 2004.

[11] N. O. Sokal, A. D. Sokal, "Class E-A New Class of High-Efficiency Tuned Single-Ended Switching Power Amplifiers," IEEE Journal of Solid-State Circuit, vol. SC-10, no 3, p. 168-176, 1975.

[12] F. H. Raab, "Idealised Operation of the Class E Tuned Power Amplifier," IEEE Transactions on Circuit and Systems, Vol.Cas-24, no 12, p.725-735, 1977.

[13] F. H. Raab, "Effects of Variation on the Class E Tuned Power Amplifier," IEEE Transactions on Circuit and Systems, Vol.Sc -16, no 2, p.239-247, 1978.

[14] S. C. Ripps, RF Amplifier for Wireless Communication, Artech House, London, p.337, 1999.

[15] A. Mihai, $R F$ Power Amplifiers, Noble Publishing Corporation, Atlanta, 366 p., 2001.

[16] D. C. Galbraith, R. L. Soma, "A wide-band Efficient Inductive Trandermal Power and Data Link with Coupling Insensitive Gain," IEEE on Biomedical engineering, Stand ford, Vol. BME-34, no 4, p.266275 , avril 1987

[17] E. Tunstel, M. Oliveira, S. Bergan, "Fuzzy Behavior Hierarchies for Multi-Robot Control," International Journal of Intelligent Systems, vol. 17, p. 449-470, 2002.

[18] V. Torra, "A Review of the Construction of Hierarchical Fuzzy Sytems," International Journal of Intelligent Systems, vol. 17, p.531-543, 2002.

[19] H. Yavuz, A. Bradshaw, "A New Conceptual Approach to the Design of Hybrid Control Architecture for Autonomous Mobile Robots, " Journal of Intelligent and Robotic Systems, 34, p.1-26, 2002.

[20] R.-A. Brooks, "A Robust Layered Control System for a Mobile Robot," M.I.T. Artificial Intelligence Laboratory, p.1-25, september 1985.

[21] R.-A. Brooks, "Intelligence without Representation," Artificial Intelligence, vol. 47, p.139-159, 1991.

[22] A. Saffiotti, "The Uses of Fuzzy Logic in Autonomous Robot Navigation," Soft Computing, vol. 1, p.180-197, 1997. 